Nota

\title{
VARIAÇÃO DE GRAUS-DIA EM PIRACICABA (SP), EM ANOS DE EL NIÑO E LA NIÑA $\left(^{(1)}\right.$
}

\author{
ANGÉLICA PRELA $\left(\left(^{2 *}\right)\right.$; BEATRIZ IBET LOZADA GARCIA $\left({ }^{3}\right)$; ANTONIO ROBERTO PEREIRA $\left({ }^{4}\right)$
}

\begin{abstract}
RESUMO
Foi estudada a relação entre El Niño Oscilação Sul (ENOS) e graus-dia (GD) para Piracicaba (SP), no período de 1950 a 2001. Quando se avaliou GD em anos de ocorrência de El Niño, utilizando valores médios, observou-se desvios principalmente, positivos e em anos de ocorrência de La Niña, desvios positivos no $1 .^{\circ}$ semestre e negativos no $2{ }^{\circ}$ semestre. No entanto, quando se analisou GD em anos isolados de ocorrência de cada fenômeno, observou-se variação nos resultados de acordo com ano e intensidade do fenômeno. Não foi observada influência generalizada de ENOS em relação a GD.
\end{abstract}

Palavras-chave: graus-dia, temperatura, ENOS

\section{ABSTRACT \\ VARATION IN THE DEGREE-DAYS IN PIRACICABA (SP), BRAZIL, IN YEARS OF EL NIÑO AND LA NIÑA}

The relation between El Niño Souther Oscillation (ENSO) and degree-day (GD) was studied for Piracicaba (SP), for the period from 1950 to 2001. When GD in years of occurrence of El Niño was evaluated, using mean values, was observed mainly positives deviations and in years of occurrence of La Niña, was observed positive desvios in the first semester and negatives in the second semester. However, when GD in isolated years of occurrence of each phenomenon was analyzed, observed variation in the results in accordance with year and intensity of the phenomenon. Generalized influence of ENOS in relation to the GD was not observed.

Key words: degree-days, temperature, ENSO.

$\left({ }^{1}\right)$ Recebido para publicação em 26 de outubro de 2005 e aceito em 16 de maio de 2006.

( $\left.{ }^{2}\right)$ Apta Regional Médio Paranapanema - Caixa Postal 263, 19800-000 Assis (SP). E-mail: angelica@aptaregional.sp.gov.br. *Autor correspondente.

$\left({ }^{3}\right)$ Instituto Nacional de Investigaciones Agrícolas - Venezuela - Táchira. E-mail: blozada@inia.gob.ve

( $\left.{ }^{4}\right)$ Departamento Ciências Exatas- LCE, Esalq-USP. Caixa Postal 9, 13418-970 Piracicaba (SP). E-mail: arpereir@esalq.usp.br. 


\section{INTRODUÇÃO}

Os efeitos dos fenômenos El Niño (EN) e La Niña (LN) são observados em escala global e alteram principalmente o regime pluviométrico e térmico em diversas regiões. Segundo Berlato e Fontana (2003) na Região Sudeste observa-se um aumento moderado da temperatura do ar durante os eventos EN, e durante os eventos de LN as temperaturas no inverno e no verão são próximas ou abaixo da média climatológica.

No caso de Piracicaba, Marin et al. (1999) concluíram que a temperatura anual média foi acima do normal em 56\% dos anos com eventos. DetTinger et al. (2001) concluíram que no Brasil os anos EN estão associados a temperaturas mais altas. Por sua parte, Grimn e Togatlian (2002) especificam que as perturbações mais consistentes da temperatura de superfície durante os eventos EN e LN ocorrem durante o inverno (junho, julho e agosto); temperaturas acima do normal ocorrem durante os eventos EN e abaixo do normal durante eventos LN.

Um dos elementos envolvido no estudo da relação clima-vegetal é a temperatura do ar, considerando-se a época de ocorrência dos subperíodos específicos de cada cultura e da semeadura. Para se obter o maior aproveitamento da disponibilidade climática em uma região, é necessário que a semeadura seja realizada em datas que proporcionem a coincidência dos períodos críticos da cultura com aquelas cujas restrições climáticas não sejam relevantes. (BARBANO, et al., 2001).

O método de graus-dia tem sido empregado para se determinar a duração dos subperíodos e do ciclo total de uma cultura no campo, já que a relação entre a temperatura e a taxa de desenvolvimento relativo da planta é considerada linear. No entanto, possuem limitação quando existem condições de deficiência hídrica no solo (Prela e Ribeiro, 2000) ou temperaturas extremas. As taxas de muitos processos do desenvolvimento das plantas e do sincronismo dos estádios fenológicos são fortemente dependentes da temperatura (JONES, 1992).

Segundo BARBANo et al. (2001), a temperatura do ar influencia os processos fisiológicos dos vegetais, interferindo em cada subperíodo do ciclo dos vegetais. A temperatura do ar é a principal causa de variação do número de dias do ciclo vegetativo.

Locais e condições climáticas favoráveis e épocas de plantio adequadas devem ser escolhidas antecipadamente para se evitar queda de produtividade e garantir a produção desejável. Os graus-dia podem ser utilizados por produtores e indústrias de processamento de produtos principalmente hortícolas, podendo prever a data de colheita e realizar o planejamento da semeadura.

O objetivo deste estudo é relacionar os índices de graus-dia e o fenômeno ENOS para o município de Piracicaba (SP) e analisar a variação e as épocas de ocorrência.

\section{MATERIAL E MÉTODOS}

Foram utilizadas séries de dados de temperatura máxima e mínima para o período de 51 anos (1950 a 2001) do posto meteorológico, localizado na ESALQ-USP, em Piracicaba, SP (Latitude: $22^{\circ} 42^{\prime}$ 30" Sul - Longitude de $47^{\circ} 38^{\prime}$ 00" Oeste - Altitude de 546 metros).

As normais climatológicas do local foram calculadas utilizando os dados dos anos em que não ocorreram os fenômeno, chamados anos neutros. Também foram calculados separadamente os graus-dia para os anos com ocorrência de El Niño e de La Niña.

Tabela 1. Anos de ocorrência do fenômeno ENOS

\begin{tabular}{|c|c|c|c|c|c|}
\hline Ano/Período & Evento & Ano/Período & Evento & Ano/Período & Evento \\
\hline 1949-1951 & La Niña & 1970-1971 & La Niña & 1986-1988 & El Niño \\
\hline 1951 & El Niño & 1972-1973 & El Niño & 1988-1989 & La Niña \\
\hline 1953 & El Niño & $1973-1976$ & La Niña & 1990-1993 & El Niño \\
\hline 1954-1956 & La Niña & 1976-1977 & El Niño & 1994-1995 & El Niño \\
\hline 1957-1959 & El Niño & 1977-1978 & El Niño & 1995-1996 & La Niña \\
\hline 1963 & El Niño & 1979-1980 & El Niño & 1997-1998 & El Niño \\
\hline 1964-1965 & La Niña & 1982-1983 & El Niño & 1998-2001 & La Niña \\
\hline 1965-1966 & El Niño & 1983-1984 & La Niña & - & - \\
\hline 1968-1970 & El Niño & 1984-1985 & La Niña & - & - \\
\hline
\end{tabular}

Fonte: Climate Prediction Center (CPC)/NCEP/NOAA - Estados Unidos. 
Para o cálculo de graus-dia (GD em $\left.{ }^{\circ} \mathrm{C} . \mathrm{d}\right)$ tomou-se como temperatura base $\left(\mathrm{Tb}\right.$ em $\left.{ }^{\circ} \mathrm{C}\right)$ igual a $6^{\circ} \mathrm{C}$, pois a maioria das espécies agrícolas iniciam seu crescimento a partir dessa temperatura, chamada Zero Vital ( MотA et al., 1981).

Foi utilizada a seguinte equação: $G D=((T x+$ $\mathrm{Tn}) / 2-\mathrm{Tb})$

Sendo: $\mathrm{Tx}=$ temperatura máxima; $\mathrm{Tn}=$ temperatura mínima; $\mathrm{Tb}=$ temperatura basal mínima

\section{RESULTADOS E DISCUSSÃO}

Verificou-se que para os anos de ocorrência de El Niño houve uma redução nos graus-dia em abril, agosto, setembro e outubro, indicando ocorrência de temperaturas abaixo das registradas em anos neutros. Nos demais meses (janeiro, fevereiro, março, maio, junho, julho, novembro e dezembro) observou- se um desvio positivo, indicando aumento da temperatura (Tabela 2, Figura 2).

Em anos de ocorrência de El Niño, são observados desvios positivos em fevereiro e julho, e em setembro e outubro, os desvios negativos mais acentuados (Figura 1).

Em relação à La Niña, os desvios positivos foram observados de janeiro a junho, e a partir de julho, até dezembro, observou-se um desvio negativo, corroborando com a literatura (BERLATO e FONTANA, 2003; Grimn e TogatLian, 2002) na qual em anos de La Niña, a temperatura tende a ser mais baixa a partir de julho. Os maiores desvios foram observados em agosto, setembro, outubro e novembro. Os desvios observados em julho e dezembro são menores, o que pode estar relacionado com o inicio e fim da influência do fenômeno. Nos meses em que os desvios foram positivos, observou-se pouca variação nos graus-dia médios (Figura 2).

Tabela 2. Graus-dia médios e desvio-padrão para anos normais, El Niño e La Nina

\begin{tabular}{lccccc}
\hline Meses & Normais & El Niño & Desvio padrão & La Niña & Desvio-padrão \\
\hline Janeiro & 18.6 & 18.9 & 0.25 & 18.7 & 0.1 \\
Fevereiro & 18.0 & 19.4 & 1.03 & 18.1 & 0.1 \\
Março & 17.9 & 18.7 & 0.64 & 17.6 & 0.2 \\
Abril & 15.9 & 15.3 & $-0.39^{*}$ & 15.9 & 0.0 \\
Maio & 12.4 & 12.8 & 0.32 & 12.5 & 0.1 \\
Junho & 11.3 & 11.6 & 0.19 & 11.6 & 0.2 \\
Julho & 11.7 & 12.6 & 0.71 & 11.5 & $-0.1^{*}$ \\
Agosto & 14.0 & 13.2 & $-0.57^{*}$ & 13.2 & $-0.6^{*}$ \\
Setembro & 16.6 & 15.0 & $-1.07^{*}$ & 15.4 & $-0.8^{*}$ \\
Outubro & 17.9 & 16.2 & $-1.17^{*}$ & 15.5 & $-1.6^{*}$ \\
Novembro & 17.1 & 17.6 & 0.32 & 15.7 & $-1.0^{*}$ \\
Dezembro & 18.0 & 18.1 & 0.05 & 17.3 & $-0.5^{*}$ \\
\hline
\end{tabular}

*Desvios negativos indicam redução nos graus-dia em relação aos anos normais.

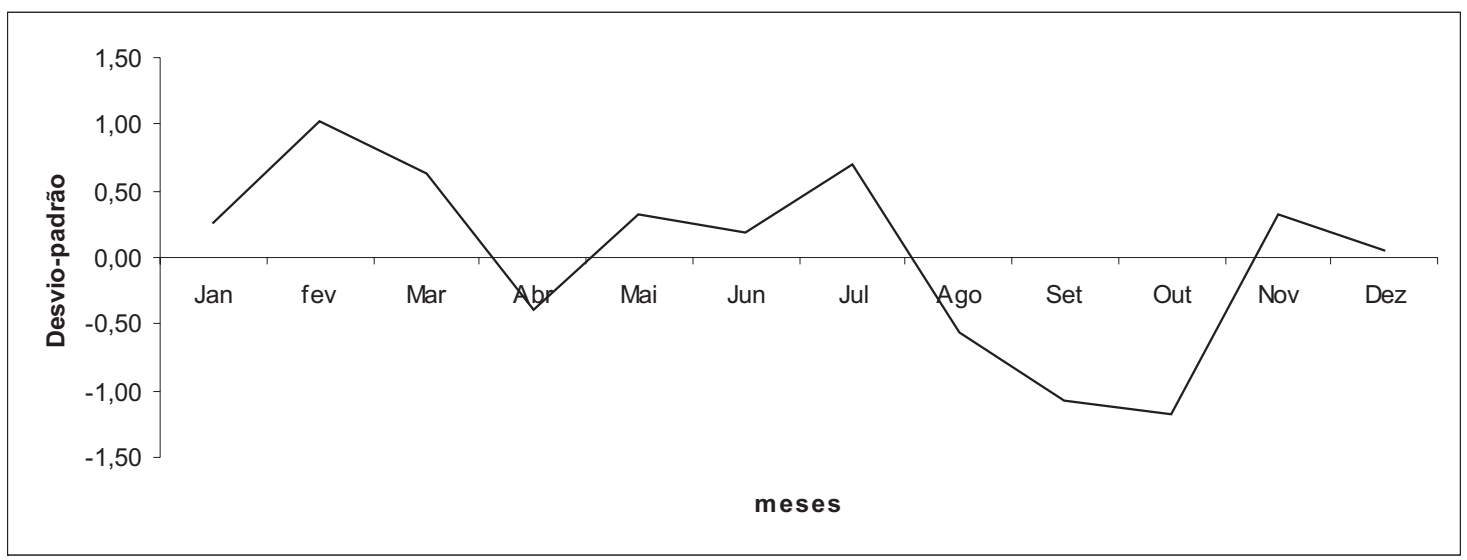

Figura 1. Desvio-padrão entre anos Normais e anos de ocorrência de El Niño. 


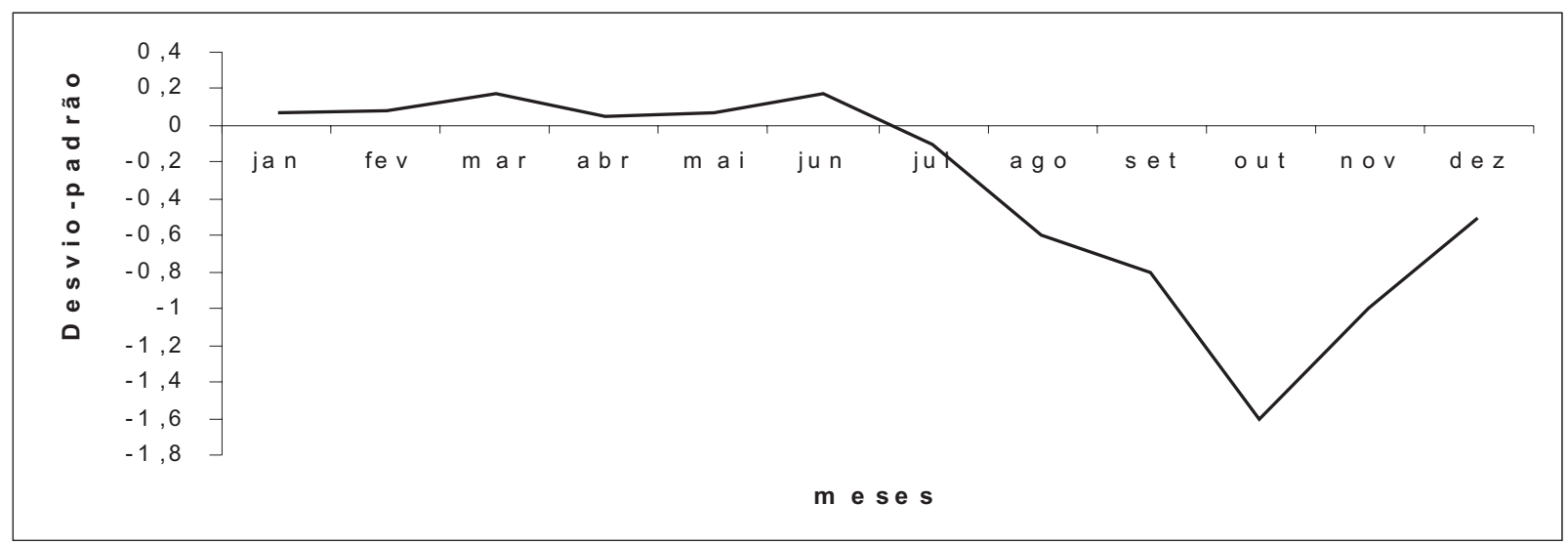

Figura 2. Desvio-padrão médio entre anos normais e anos de La Niña.

Considerando o número de eventos El Niño e La Niña, no período analisado (1950 a 2001), temos 15 e 10 eventos respectivamente. Alguns eventos, se prolongaram por mais de um ano, podendo chegar até três anos consecutivos; assim, para a análise realizada (Tabela 3) considerou-se o número de anos em que houve a ocorrência do fenômeno em determinado mês.

Em anos com ocorrência de La Niña, observouse maior número de eventos com influência negativa, corroborando com dados de variação de graus-dia médios acumulados, com exceção de alguns meses. Em setembro, por exemplo, observou-se durante a ocorrência de La Niña, em $65 \%$ dos casos, um aumento nos graus-dia médios mensais, indicando aumento de temperatura nesse período; no entanto, nesse mês houve desvio negativo. Este fato foi decorrente de que embora houvesse maior número de desvios positivos, os negativos foram mais acentuados, em torno de -3 a $-4{ }^{\circ} \mathrm{C}$. O inverso ocorreu em janeiro, maio e junho, e em anos com ocorrência de El Nino, observou-se esse mesmo fenômeno em agosto, setembro e outubro.

Em função das diferenças observadas nas análises anteriores, verificou-se que essas variam de acordo com a intensidade de fenômeno. Assim, analisou-se a variação de graus-dia acumulados, em anos particulares, dentro do período estudado, com ocorrência de La Niña e El Niño, classificados em diferentes intensidades (fraco, moderado e forte), e comparou-se com graus-dia médios de anos neutros.

Tabela 3. Análise pontual mensal, da porcentagem de ocorrência de desvios positivos e negativos, em anos de El Niño e La Niña, durante o período de 1950 a 2001

\begin{tabular}{|c|c|c|c|c|}
\hline \multirow{2}{*}{ Meses } & \multicolumn{2}{|c|}{ La Niña } & \multicolumn{2}{|c|}{ El Niño } \\
\hline & Positivo & Negativo & Positivo & Negativo \\
\hline & & 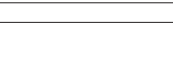 & & \\
\hline Janeiro & 44 & 66 & 65 & 35 \\
\hline Fevereiro & 66 & 44 & 54 & 46 \\
\hline Março & 61 & 39 & 54 & 46 \\
\hline Abril & 61 & 39 & 46 & 54 \\
\hline Maio & 36 & 64 & 68 & 32 \\
\hline Junho & 38 & 62 & 50 & 50 \\
\hline Julho & 40 & 60 & 57 & 43 \\
\hline Agosto & 25 & 75 & 54 & 46 \\
\hline Setembro & 65 & 35 & 57 & 43 \\
\hline Outubro & 40 & 75 & 51 & 49 \\
\hline Novembro & 18 & 82 & 32 & 68 \\
\hline Dezembro & 44 & 66 & 62.5 & 37.5 \\
\hline
\end{tabular}


No ano em que o evento El Niño foi considerado forte (1997), ocorreu aumento dos grausdia acumulados, a partir de julho, com leve queda em outubro. Nos demais meses, os graus-dia acumulados foram semelhantes às médias mensais dos anos neutros. Essa situação não coincide com resultados das análises dos desvios e da literatura em geral, cujos desvios positivos em anos de ocorrência de El Nino são observados no primeiro semestre. Em um ano de ocorrência considerado moderado (1994), observou-se que durante a maior parte desse ano os graus-dia acumulados permaneceram acima da média dos anos neutros, com exceção em março e junho, quando esses valores se igualaram. A mesma tendência foi observada em um ano de ocorrência fraca de El Niño (1977); nesse ano, os valores de graus-dia acumulados em abril, maio e dezembro ficaram abaixo das médias mensais dos anos neutros (Figura 3).

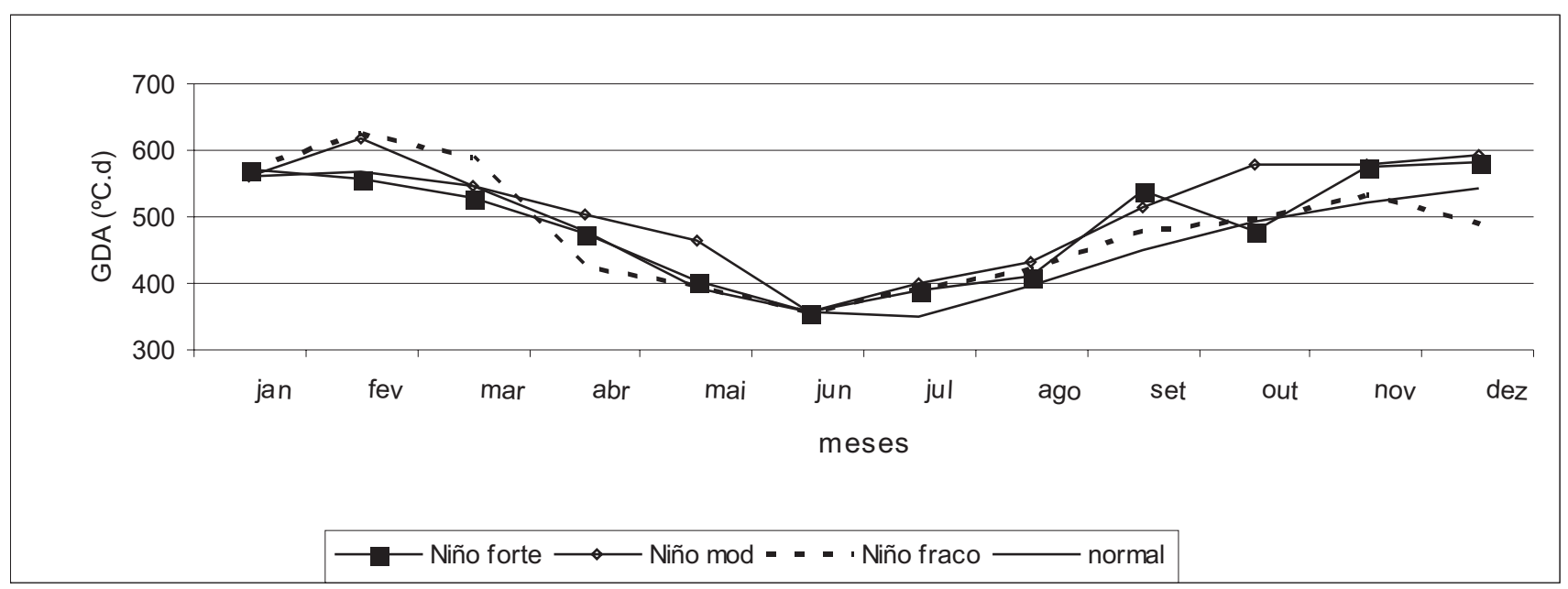

Figura 3. Graus-dia acumulados (GDA) mensais em ano neutro e anos com ocorrência de El Niño considerados forte (1997), moderado (1994) e fraco (1977) ocorridos entre 1950 e 2001.

Analisando os eventos de La Niña, verificou-se que em anos de ocorrência do fenômeno forte e moderado, os graus-dia acumulados ficaram abaixo da média de anos neutros, de abril a agosto, que corresponde ao mesmo período descrito na literatura. No entanto, em 1984, ano em que o evento foi considerado fraco, os graus-dia acumulados foram superiores às médias de anos neutros, durante todo o ano, com exceção de setembro, quando se registraram valores inferiores (Figura 4).

Quando se observam as figuras 3 e 4, verificase que cada evento deve ser considerado como situação particular em relação às anomalias de grausdia acumulados. Os dados quando analisados por médias, mascaram os dados pontuais; e quando analisados cada ano em particular, observam-se comportamentos diferentes. Esse fato revela que cada evento tem uma situação particular nas anomalias de temperatura.

Não foi observada influência generalizada do fenômeno ENOS, para Piracicaba, em relação à temperatura e aos graus-dia acumulados, pois a influência pode variar de acordo com a intensidade e o ano de ocorrência do fenômeno. A análise de graus-dia acumulados, quando feita ano a ano, evidencia influências diferentes.

\section{REFERÊNCIAS}

BARBANO, M.T.; SAWAZAKI, E.; BRUNINI, O.;GALLO, P.B.; PAULO, E.M. Temperatura-base e acumulo térmico no subperíodo emergência-florescimento masculino em cultivares de milho no Estado de São Paulo. Revista Brasileira de Agrometeorologia, Santa Maria, v.9, n.2, p. 261-268, 2001.

BERLATO, M.A.; FONTANA, D.C. El Niño e La Niña: impactos no clima, na vegetação e na agricultura do Rio Grande do Sul; aplicações de previsões climáticas na agricultura. Porto Alegre: UFRGS, 2003. $110 \mathrm{p}$.

DETTINGER, M.D.; BATTISTI D.S.; MCCABE, G.J.; GARREAUD, R.D.; BITZ C.M. Interhemipheric effects of interannual and decadal ENSO-Like climate variations on the Americas. In: Present and Past Interhemispheric climate linkages in the Americas and the societal effects. London: Academic Pess, 2001. 
GRIM, A.M.; TOGATLIAN, I. M de. Relação entre eventos EL NIÑO/LA NIÑA e freqüência de ocorrência de extremos frios e quentes de temperatura no Cone Sul da América do Sul. In: CONGRESSO BRASILEIRO DE METEOROLOGIA. Foz de Iguaçu, PR, 2002. Anais... Foz de Iguaçu: Sociedade Brasileira de Meteorologia/SBMET, 2002. p.1192-1197. CD-ROM.

JONES, H. G. Plants and microclimate: a quantitative approach to enviromental plant physiology. Cambridge: University Press, 1992. 428 p.

MARIN, F.R.; SENTELHAS, P.C.; VILLA NOVA, N.A. Influencia dos fenómenos El Niño y La Niña no clima de Piracicaba, SP. Revista Brasileira de Meteorologia, Santa Maria, v. 15, n. 1, 123-129, 2000.
MOTA, F. S. da. Meteorologia Agrícola. São Paulo, 5. ed. 1981. 376p.

PINTO, L.B.; TATSCH, J.D.; NOBLE, D.V.; MENDES, L.L.; ACOSTA, R.; CAMPOS, R.R.J. Um estudo sobre o impacto do evento La Niña 1999/2001 em Pelotas-RS. In: CONGRESSO BRASILEIRO DE METEOROLOGIA, 12., 2002 Foz do Iguaçu, Anais... Foz do Iguaçu: SBA, 2002, p.1152-1153.

PRELA, A.; RIBEIRO, A.M.A. Determinação de grausdia para o amendoinzeiro no subperíodo semeadura colheita. Revista Brasileira de Agrometeorologia, Santa Maria, v.2, n.8, p.321-324, 2000. 